\title{
Cognitive characteristics of PTEN hamartoma tumor syndromes
}

\author{
Robyn M. Busch, PhD ${ }^{1}$, Jessica S. Chapin, $\mathrm{PhD}^{1,6}$, Jessica Mester, MS',3, Lisa Ferguson, MA ${ }^{1}$, \\ Jennifer S. Haut, PhD', Thomas W. Frazier, $\mathrm{PhD}^{2,4}$ and Charis Eng, MD, PhD $2,3,5$
}

Purpose: We sought to characterize cognition in individuals with germline PTEN mutations $(n=23)$ as well as in PTEN mutationnegative individuals with classic Cowden syndrome or BannayanRiley-Ruvalcaba syndrome $(n=2)$.

Methods: Twenty-five individuals completed a comprehensive neuropsychological evaluation. One sample $t$-tests and effect sizes were used to examine differences in participant test scores compared with normal controls. Composite scores were created for each patient within each of the cognitive domains assessed and classified as above average, average, or below average according to normative standards. $\chi^{2}$ analyses compared these classifications to expected proportions in normal control samples.

Results: The mean intelligence quotient was in the average range, and the range of intellectual functioning was very wide (from extremely low to very superior). However, in a large subset of patients, scores were lower than expected on measures of motor functioning, executive functioning, and memory recall, suggesting disruption of frontal circuits in these participants.

Conclusion: This is the first study to characterize cognition in individuals with PTEN mutations and associated syndromes using a comprehensive neuropsychological battery. Contrary to previous reports suggesting an association with intellectual disability, the mean intelligence quotient was average, and there was a broad range of intellectual abilities. Specific evidence of disruption of frontal circuits may have implications for treatment compliance and cancer surveillance, and further investigation is warranted.

Genet Med 2013:15(7):548-553

Key Words: Bannayan-Riley-Ruvalcaba syndrome; cognition; Cowden syndrome; neuropsychology; PTEN; PTEN hamartoma tumor syndromes

\section{INTRODUCTION}

PTEN (OMIM no.+601728), is a major tumor suppressor gene located on 10q23.3 with a documented role in heritable and sporadic malignancies. Germline PTEN mutations have been found to occur in a subset of several seemingly disparate clinical syndromes including Cowden syndrome (CS, OMIM no. 158350) and Bannayan-Riley-Ruvalcaba syndrome (BRRS, OMIM no. 153480) (reviewed in Zbuk and Eng, 2007; ref. 1). Irrespective of the specific syndrome, individuals carrying germline PTEN mutations are grouped under the umbrella term "PTEN hamartoma tumor syndrome" (PHTS). ${ }^{1,2} \mathrm{CS}$ is an autosomal dominant disorder characterized by multiple hamartomas and a high risk of malignancies, with increased lifetime risks for female breast cancer (85\%), epithelial thyroid cancer (35\%), endometrial cancer $(28 \%)$, renal cancer (34\%), colorectal cancer (9\%), and melanoma (6\%). ${ }^{3}$ Although the risks of developing neoplasms have recently been reasonably well characterized, the neurobehavioral phenotype remains unknown.

Despite the findings from family studies and small case studies that developmental delay, intellectual disability, and central nervous system anomalies are associated with PHTS and related syndromes, ${ }^{4-12}$ there are no studies that have systematically investigated the cognitive characteristics of these patients.
The goal of this exploratory study was to characterize cognition in individuals with known PTEN mutations as well as in individuals with classic CS and BRRS who do not have PTEN mutations.

\section{Participants}

\section{MATERIALS AND METHODS}

Participants were recruited from an ongoing prospective observational study of PHTS, and consisted of individuals with Cowden and Cowden-like syndromes. Eligible participants were informed about the current cognitive study and invited to participate if they met the following inclusion criteria: (i) had undergone PTEN mutation analysis leading to the detection of a pathogenic mutation, either by Cleveland Clinic research or clinical laboratories $(n=23)$ or, (ii) in the absence of a deleterious PTEN mutation, had presented with strong phenotypic features consistent with a diagnosis of CS or BRRS as outlined for $\mathrm{CS}$ by the International Cowden Consortium ${ }^{13}$ and for BRRS by disease-specific expert consensus ${ }^{14}(n=2)$. All research participants were required to travel to Cleveland, Ohio, for participation in the study.

A total of 25 research participants (20 adults, 5 children) were prospectively enrolled in this institutional review

${ }^{1}$ Neurological Institute, Cleveland Clinic, Cleveland, Ohio, USA; ${ }^{2}$ Genomic Medicine Institute, Cleveland Clinic, Cleveland, Ohio, USA; ${ }^{3}$ Taussig Cancer Institute, Cleveland Clinic, Cleveland, Ohio, USA; ${ }^{4}$ Pediatric Institute, Cleveland Clinic, Cleveland, Ohio, USA; ${ }^{5}$ Department of Genetics and Genome Sciences, Case Western Reserve University, Cleveland, Ohio, USA; ${ }^{6}$ Current affiliation: Center for Neuropsychology, Aurora Health Care, Grafton, Wisconsin, USA. Correspondence: Robyn M. Busch (buschr@ccf.org) 
board-approved study (IRB no. 07-289) between July 2007 and July 2012. Informed consent was obtained from each of the adult participants and parental consent was obtained for child participants, in addition to assent from the child. A neuropsychological assessment was conducted in all the participants as part of the study. The adult participants ranged in age from 23 to 60 years (mean $=43.95, \mathrm{SD}=12.19)$ and had a mean education duration of $16.25(\mathrm{SD}=2.36)$ years. The five children in the study were of ages $5,5,14,16$, and 17 years. For the 5-year-olds, given that they were very young, the battery of cognitive tests administered did not include assessment of all cognitive domains. PTEN mutations were detected in 23 of the participants. Of the two mutation-negative participants, one had classic BRRS and one had classic CS. Given that most of the participants in the study had PTEN mutations (92\%), this series will hereinafter be referred to as PHTS. The demographic and health characteristics of the participants are summarized in Table 1, and the detailed phenotypic features, including Cleveland Clinic PTEN risk score ${ }^{15}$ are described in Supplementary Table S1 online.

Four of the participants had a history of brain surgery. Two of these had undergone resection of cerebellar tumors (one with confirmed Lhermitte-Duclos disease (LDD) and one with probable LDD), one had undergone surgery and ventriculoperitoneal shunt placement for a brain stem meningioma with hydrocephalus, and one had undergone surgery and ventriculoperitoneal shunt placement for an arachnoid cyst. Brain magnetic resonance imaging (MRI) scans were completed on an additional 10 participants as part of their clinical care, and were available for review. In these, the most common findings were venous anomaly/angioma, Chiari I malformation, and cystic lesion. See Table 1 and Supplementary Table S1 online for more information.

Fourteen of the participants had a history of cancer not involving the central nervous system, for which they underwent surgery. In addition, two participants completed chemotherapy, two underwent radiation, and three participants had both chemotherapy and radiation. The cancer types and treatments are outlined in Table 1.

\section{Genetic analyses}

Germline DNA was extracted in the Genomic Medicine Institute's Genomic Medicine Biorepository and analyzed in a research laboratory (C.E.) for PTEN denaturing gradient gel electrophoresis or Lightscanner-based mutational scanning ( $99 \%$ sensitive in Eng laboratory). Samples with variations seen in mutation scanning were re-assayed using PCR and Sanger sequencing in the Genomics Core Facility, Cleveland Clinic. Large deletions and rearrangements were detected by multiplex ligation-dependent probe amplification and confirmed by quantitative PCR.

\section{Measures}

All the study participants completed a neuropsychological assessment that included clinical and structured diagnostic
Table 1 Demographic and health data of participants

\begin{tabular}{lcc} 
& Range & Mean (SD) \\
\hline Age & & \\
Children $(n=5)$ & $5-17$ & $11.40(5.94)$ \\
Adults $(n=20)$ & $23-60$ & $43.95(12.19)$ \\
Education & & \\
Adults $(n=20)$ & $12-21$ & $16.25(2.36)$ \\
FSIQ $(n=24)$ & $65-135$ & $103.21(17.17)$ \\
& Number & Percent \\
Sex & & \\
Male & 7 & $28 \%$ \\
Female & 18 & $72 \%$ \\
PTEN mutation status & & \\
Positive & 23 & $82 \%$ \\
Negative & 2 & \\
$\quad$ Phenotype \\
$\quad$ Cowden syndrome \\
BRRS
\end{tabular}

Brain MRI findings ${ }^{\mathrm{a}}$

Negative/nonspecific findings

Venous anomoly/angioma only

+Cystic lesion

Lhermitte-Duclos disease ${ }^{b}$

Chiari I malformation only

+ Cystic lesion $^{\mathrm{b}}$

Meningioma $^{b}$

Perinatal ischemia

Prior brain surgery/resection

Cerebellar tumor

Arachnoid cyst

Meningioma

Cancer history

Breast only

Thyroid only

Breast and thyroid

Breast and other cancer(s) ${ }^{c}$

Thyroid and other cancer(s) ${ }^{d}$

Other cancer(s)e

Cancer treatments ${ }^{f}$

Surgery only

+Chemotherapy only

+Radiation only

$21 \%$

1

2

$2 \quad 15 \%$

$2 \quad 21 \%$

1

2

1

$15 \%$

$7 \%$

$16 \%$

2

1

1

14

4

+Chemotherapy and radiation

BRRS, Bannayan-Riley-Ruvalcaba syndrome; FSIQ, full-scale intelligence quotient; MRI, magnetic resonance imaging.

aResults of brain MRI were available for 14 participants—reported percentages relate to these participants only. ${ }^{\mathrm{b}} \mathrm{C}$ ounts include participants who had undergone prior brain surgery/resection. 'Uterine, skin, colorectal, and/or salivary. 'Uterine and/ or skin. eProstate, lung, and skin. 'Results are reported only for participants who had been diagnosed with cancer. 
interviews conducted by a board-certified neuropsychologist (R.M.B., J.S.C., or J.S.H.) as well as a comprehensive battery of cognitive tests that included measures of intellectual functioning, attention/concentration, visuomotor processing speed, language, executive functioning (EF), visuospatial skills, memory recall, memory recognition, academic skills, and motor functioning. See Supplementary Tables S2 and S3 online for a list of cognitive measures included in the neuropsychological assessment. All measures were scored according to their published test manuals, using age-corrected and, when appropriate, education-corrected norms. To allow for comparisons across measures, all test scores were transformed to standard scores with a mean of 100 and a SD of 15 . Higher standard scores reflect better performance. A summary of the cognitive data is provided in Supplementary Table S2 online.

\section{Analyses}

Composite scores were created for each patient within each of the cognitive domains assessed (e.g., language, EF) by totaling the standard scores of the completed measures within the cognitive domain and dividing the sum by the total number of measures within that domain. See Supplementary Table S3 online for a list of the individual cognitive measures included in each domain.

One-sample $t$-tests examined differences between test scores from individuals with PHTS and normative data obtained from control samples in published test manuals (normative mean standard score $=100, \mathrm{SD}=15$ ) on domain composites as well as on the individual subtests within each domain. Next, each participant's domain scores were categorized as above average, average, or below average, using a $1 \mathrm{SD}$ (i.e., \pm 15 points) cutoff from the mean (i.e., 100). $\chi^{2}$ Analyses with exact tests were then carried out for each cognitive domain to determine whether the proportion of participants within each of the three test performance categories differed significantly from expected proportions in the normal population (i.e., $16 \%$ above average, $68 \%$ average, and $16 \%$ below average). Identical subanalyses were performed on the individual measures within each cognitive domain.

\section{RESULTS}

One-sample $t$-tests revealed that mean scores among individuals with PHTS were significantly lower than those of control samples in the motor $(\mathrm{t}(22)=-5.02, P<0.001, d=-0.94)$ and $\mathrm{EF}(\mathrm{t}(22)=-3.67, P=0.001, d=-0.70)$ domains, with large and medium effect sizes, respectively. There was also a similar trend in the memory recall domain $(\mathrm{t}(24)=-1.80, P=0.085, d=$ -0.38 ), albeit with a smaller effect size. No significant differences were observed between individuals with PHTS and normal controls on any of the other cognitive domain scores (Table 2).

$\chi^{2}$ analyses with exact tests revealed that more individuals with PHTS had reduced performance in the domain of motor functioning $\left(\chi^{2}(2)=9.41, P=0.009\right)$ as compared with expected proportions in normal controls. There were also similar trends in memory recall $\left(\chi^{2}(2)=5.24, P=0.068\right)$ and $\mathrm{EF}\left(\chi^{2}(2)=4.72\right.$, $P=0.097)$. The proportion of PHTS individuals within each of
Table 2 Mean (SD) scores of PHTS subjects on neuropsychological domains and magnitude (Cohen's $d$ ) of difference from the scores of the normal population

\begin{tabular}{|c|c|c|c|}
\hline \multirow[b]{2}{*}{ Domain } & \multirow{2}{*}{$\begin{array}{c}\text { PHTS } \\
\text { Mean (SD) }\end{array}$} & \multicolumn{2}{|c|}{$\begin{array}{l}\text { PHTS vs. population } \\
\text { controls }\end{array}$} \\
\hline & & $\mathrm{t}(P)$ & Cohen's $d$ \\
\hline Intelligence & $103.2(17.2)$ & $0.92(0.370)$ & 0.20 \\
\hline Attention/working memory & $102.6(16.4)$ & $0.73(0.474)$ & 0.17 \\
\hline Processing speed & $98.8(12.9)$ & $-0.46(0.651)$ & -0.09 \\
\hline Language & $97.1(15.3)$ & $-0.95(0.351)$ & -0.19 \\
\hline Executive functioning & $90.3(12.7)$ & $-3.67(0.001)$ & -0.70 \\
\hline Visuospatial & $97.9(15.6)$ & $-0.66(0.514)$ & -0.14 \\
\hline Memory recall & $94.0(16.7)$ & $-1.800(0.085)$ & -0.38 \\
\hline Memory recognition & $100.2(14.7)$ & $0.08(0.935)$ & 0.01 \\
\hline Academic skills & $104.6(14.4)$ & $1.53(0.140)$ & 0.31 \\
\hline Motor & $87.2(12.2)$ & $-5.02(<0.001)$ & -0.94 \\
\hline
\end{tabular}

PHTS subjects show large, significant differences in respect of the motor and executive functioning domains. Population controls; mean $=100, S D=15$. Boldface values reflect those effects' sizes in the medium to large range. PHTS, PTEN hamartoma tumor syndrome.

the three performance groups did not differ significantly from that of normal controls in any of the other cognitive domains that were assessed (Figure 1).

When individual tests within the motor functioning composite were examined, a larger proportion of individuals with PHTS were found to have lower fine manual dexterity as compared with normal controls (grooved pegboard test, $P$ $=0.000$ ). Of note, seven participants demonstrated reduced performance with only one hand, whereas 10 participants demonstrated reduced manual dexterity bilaterally. Only six participants in this sample had bilateral manual dexterity scores within the normal range. Within the EF composite, there was a greater than expected proportion of individuals with reduced performance on a measure of phonemic verbal fluency (Controlled Oral Word Association Test, $P=.002$ ), and a trend in this direction on a novel problemsolving task (Wisconsin Card Sorting Test, Perseverative Errors, $P=0.094)$. Finally, examination of measures within the memory recall composite revealed that a larger percentage of individuals had lower performance on the immediate recall trials of a word-list learning task (Rey Auditory Verbal Learning Test, Immediate Recall, $P=0.009$ ), and that there was a trend in this direction on the delayed recall trial of this measure $(P=0.059)$. See Table 2, Supplementary Tables S2 and $\mathbf{S 3}$ online for a summary of results on all measures.

An examination of the overall cognitive profiles of each individual in this study revealed that three of the participants (12\%) had rather global cognitive impairments across domains, including borderline to extremely low Full Scale Intelligence Quotients (FSIQ). Two of these participants had a history of resected cerebellar tumor. The FSIQ scores of the other participants $(n=$ 22) ranged from low average to very superior (standard score: $80-135$; mean $=107.38, \mathrm{SD}=13.67)$. As mentioned earlier, the 


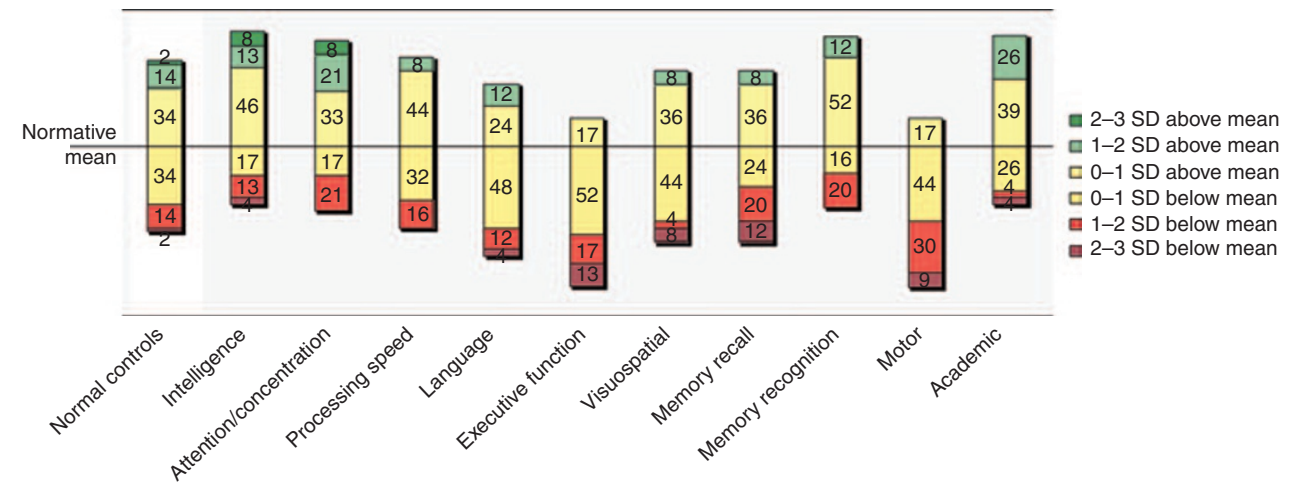

Figure 1 Cognitive performance of individuals with PHTS on composite domain scores as compared with expected proportions in normal controls. Numbers represent the percentages of patients falling within each score category. PHTS, PTEN hamartoma tumor syndrome.

two youngest children in the study, both of whom were 5 years of age, were subjected to a relatively limited battery of cognitive teats, given the limited test measures available for this age group. Therefore, cognitive profiles across all domains could not be examined for these two children. In the adolescent and adult participants with low average or better FSIQ scores $(n=20)$, the most commonly observed findings were reduced performance on measures of manual dexterity and/or EF. Specifically, seven participants (35\%) demonstrated reduced dexterity bilaterally, and five demonstrated reduced dexterity in one hand only (three in the dominant hand, two in the nondominant hand). Most of the participants demonstrated reduced performance on one $(n=$ $10)$ or two $(n=3) \mathrm{EF}$ measures. Of these, one was a participant who had undergone removal of an arachnoid cyst with placement of a ventriculoperitoneal shunt. Only one participant with low average or higher FSIQ demonstrated low performance on all four measures of EF. This patient had a history of brain stem meningioma with hydrocephalus treated with ventriculoperitoneal shunt and surgical resection. Most participants with low average or higher IQ demonstrated intact performance on measures of academic ability, memory, naming, semantic fluency, visuospatial skills, and motor speed.

Although four of the participants in this study had undergone brain surgeries/tumor resections that may have had an impact on their cognitive test performance, the overall pattern of results did not substantially change when the data were reanalyzed after excluding the data pertaining to these participants. In fact, $72 \%$ of the other adolescent/adult participants showed reduced manual dexterity in at least one hand, $89 \%$ had reduced performance on at least one measure of $\mathrm{EF}$, and $47 \%$ had reduced performance on at least one measure of memory recall. Further, the incidence of reduced performance on these tasks was not higher in participants with a history of cancer and in those who had undergone cancer treatments (i.e., chemotherapy, radiation). Therefore, the cognitive findings in this study are not driven by the effects of prior surgical or medical interventions. Although there were no obvious differences in cognitive characteristics between participants who had a PTEN mutation and those who did not, given the small sample size of mutation-negative participants $(n=2)$, it was not possible to adequately examine cognitive differences as a function of PTEN mutation. Nevertheless, when all the analyses were re-run after excluding data pertaining to the two mutation-negative participants, and the overall pattern of results remained unchanged.

\section{DISCUSSION}

This is the first study to examine cognitive functioning in individuals with PHTS and related syndromes using a comprehensive neuropsychological battery of tests to assess a wide range of cognitive domains. The existing literature suggests that there is an association between PHTS and developmental and/or intellectual disability. ${ }^{4,6,10}$ However, most studies have based their conclusions on reported developmental history (rather than on formal assessment, i.e., intelligence testing) in individuals, families, or small case series comprised primarily of children. Clearly, our study demonstrates a much wider range of intellectual capacity in individuals with PHTS than has previously been suggested. In fact, there were only three participants in our study with FSIQ scores falling within the range seen in intellectual disability (i.e., borderline to extremely low scores), and two of them had undergone brain surgery. The other participants, who constituted $88 \%$ of the study sample, had FSIQ scores in the low average to very superior range. These findings are consistent with the observations of Miles and colleagues $(1984)^{10}$ who reported that "true mental retardation was not a regular manifestation" in their sample of patients with BRRS.

The pattern of findings in this study also suggests an increased prevalence of frontal systems dysfunction in individuals with PHTS. There were a larger proportion of individuals with PHTS with reduced performance on select measures of executive, motor, and memory functioning than would be expected in the normal population. Specifically, these participants showed greater difficulty on measures of phonemic verbal fluency and fine manual dexterity and perseverative responding on a measure of novel problem-solving as compared with normal controls. Poor performances on each of these measures have been associated with disruption of frontal lobe circuits. ${ }^{16}$ Further, the memory-related difficulties observed in the participants were restricted to free-recall tasks rather than recognition memory measures. This memory pattern suggests a retrieval-based 
deficit rather than a primary encoding issue, providing further support for likely frontal systems involvement. ${ }^{17}$

Results of available MRI studies suggest a high prevalence of brain anomalies (e.g., vascular malformations, cysts, tumors) in our study participants. In fact, there were imaging abnormalities reported in $79 \%$ of the participants for whom MRI results were available. This rate may not be representative of the entire sample, given that MRI studies were conducted only in individuals in whom it was clinically indicated (i.e., $56 \%$ of this sample). Nevertheless, our findings are consistent with the few relevant imaging studies reported in the literature. ${ }^{7,18}$ Lok and colleagues $^{7}$ found cerebral abnormalities on brain MRI in 7 of 20 patients with CS who had no clinical indication of neurological dysfunction. Venous and cavernous angiomas were the most frequent abnormalities, followed by LDD; one patient had a meningioma. Although most of the observed neuroimaging abnormalities are generally considered to be "benign," it is possible that they contributed to the cognitive findings.

The overall pattern of test findings in this study is most consistent with disruption of frontal circuits; however, we cannot rule out cerebellar dysfunction as a contributing factor, given the role of the cerebellum in many aspects of motor and cognitive functioning. ${ }^{19-21}$ Dysplastic gangliocytoma of the cerebellum (i.e., LDD) is often observed in individuals with PTEN mutations and, in adults, is considered pathognomonic for CS..$^{22-24}$ At least two participants in our study had confirmed/probable LDD; however, we cannot be sure of the actual prevalence of cerebellar abnormalities in this sample, given that neuroimaging was not available for many of the participants. Future studies should include neuroimaging so as to make firm conclusions regarding the prevalence of brain anomalies, including LDD, in this population, and the potential relationship of such anomalies to cognitive functioning.

Regardless of the underlying cause of the frontal systems dysfunction observed, these cognitive difficulties may have important implications for treatment compliance and for compliance with cancer surveillance in patients with PHTS. Problems with $\mathrm{EF}$ and memory retrieval may make it difficult for patients to organize and recall information provided to them by their physicians and to remain vigilant with respect to treatment and surveillance recommendations. If future investigations provide corroborating evidence for a pattern of cognitive dysfunction primarily involving frontal circuits, cognitive rehabilitation may be indicated in at least a subset of individuals to improve treatment compliance.

A limitation of this study was that all the participants recruited were volunteers who were able to travel to Cleveland for participation in the study, and were willing to complete an $\sim 4$-hour battery of cognitive testing. It is therefore not known to what extent our sample is reflective of the larger PHTS population. Regardless of this, the results suggest that intellectual disability is less common in association with these conditions than has previously been reported. Further, there is no reason to suspect that volunteers would be more likely to display the observed pattern of frontal systems dysfunction than non-volunteers; in fact, we would hypothesize that this study captured a higherfunctioning subset of adults, given the organizational abilities needed to coordinate travel to Cleveland for participation in the study. More studies will be needed to attempt to replicate the findings of this one, to examine the potential impact of cognitive dysfunction on treatment compliance, and to determine whether cognitive rehabilitation therapies used in other patient groups with frontal systems dysfunction would be effective in a PHTS population as well.

\section{SUPPLEMENTARY MATERIAL}

Supplementary material is linked to the online version of the paper at http://www.nature.com/gim

\section{ACKNOWLEDGMENTS}

The authors express their sincere thanks to the participants in this study for volunteering their time to be a part of this research. The authors also thank Richard Naugle, PhD and Imad Najm, MD at Cleveland Clinic for providing the resources necessary to complete this work.

\section{DISCLOSURE}

C.E. is the Sondra J. and Stephen R. Hardis Endowed Chair of Cancer Genomic Medicine at the Cleveland Clinic and an ACS Clinical Research Professor. T.W.F. and C.E. are principal investigator and co-principal investigator, respectively, of a sponsored research agreement with IntegraGen to study single-nucleotide polymorphism markers in autism spectrum disorders. The other authors declare no conflict of interest.

\section{REFERENCES}

1. Zbuk KM, Eng C. Cancer phenomics: RET and PTEN as illustrative models. Nat Rev Cancer 2007;7:35-45.

2. Marsh DJ, Kum JB, Lunetta KL, et al. PTEN mutation spectrum and genotypephenotype correlations in Bannayan-Riley-Ruvalcaba syndrome suggest a single entity with Cowden syndrome. Hum Mol Genet 1999;8:1461-1472.

3. Tan MH, Mester JL, Ngeow J, Rybicki LA, Orloff MS, Eng C. Lifetime cancer risks in individuals with germline PTEN mutations. Clin Cancer Res 2012;18: 400-407.

4. Hanssen AM, Werquin H, Suys E, Fryns JP. Cowden syndrome: report of a large family with macrocephaly and increased severity of signs in subsequent generations. Clin Genet 1993;44:281-286.

5. Higginbottom MC, Schultz P. The Bannayan syndrome: an autosomal dominant disorder consisting of macrocephaly, lipomas, hemangiomas, and risk for intracranial tumors. Pediatrics 1982;69:632-634.

6. Cohen MM Jr. Mental deficiency, alterations in performance, and CNS abnormalities in overgrowth syndromes. Am J Med Genet C Semin Med Genet 2003;117C:49-56.

7. Lok C, Viseux V, Avril MF, et al.; Cancerology Group of the French Society of Dermatology. Brain magnetic resonance imaging in patients with Cowden syndrome. Medicine (Baltimore) 2005;84:129-136.

8. Longy M, Lacombe D. Cowden disease. Report of a family and review. Ann Genet 1996;39:35-42.

9. Lynch NE, Lynch SA, McMenamin J, Webb D. Bannayan-Riley-Ruvalcaba syndrome: a cause of extreme macrocephaly and neurodevelopmental delay. Arch Dis Child 2009;94:553-554.

10. Miles JH, Zonana J, Mcfarlane J, Aleck KA, Bawle E. Macrocephaly with hamartomas: Bannayan-Zonana syndrome. Am J Med Genet 1984;19: 225-234.

11. Powell BR, Budden SS, Buist NR. Dominantly inherited megalencephaly, muscle weakness, and myoliposis: a carnitine-deficient myopathy within the spectrum of the Ruvalcaba-Myhre-Smith syndrome. J Pediatr 1993;123:70-75. 
12. Starink TM, van der Veen JP, Arwert F, et al. The Cowden syndrome: a clinical and genetic study in 21 patients. Clin Genet 1986;29:222-233.

13. Pilarski R, Eng C. Will the real Cowden syndrome please stand up (again)? Expanding mutational and clinical spectra of the PTEN hamartoma tumour syndrome. J Med Genet 2004;41:323-326.

14. Gorlin RJ, Cohen MM Jr, Condon LM, Burke BA. Bannayan-Riley-Ruvalcaba syndrome. Am J Med Genet 1992;44:307-314.

15. Tan MH, Mester J, Peterson C, et al. A clinical scoring system for selection of patients for PTEN mutation testing is proposed on the basis of a prospective study of 3042 probands. Am J Hum Genet 2011;88:42-56.

16. Stuss DT, Alexander MP, Floden D, et al. Fractionation and localization of distinct frontal lobe processes: evidence from focal lesions in humans. In: Stuss DT, Knight RT (eds). Principles of Frontal Lobe Function. New York, New York: Oxford University Press, Inc., 2002:392-407.

17. Shimamura AP, Janowsky JS, Squire LR. What is the role of frontal lobe damage in memory disorders? In: Levin HS, Eisenberg HM, Benton AL (eds). Frontal Lobe Function and Dysfunction. New York, New York: Oxford University Press, Inc., 1991:173-195
18. Tan WH, Baris HN, Burrows PE, et al. The spectrum of vascular anomalies in patients with PTEN mutations: implications for diagnosis and management. J Med Genet 2007;44:594-602.

19. O'Halloran CJ, Kinsella GJ, Storey E. The cerebellum and neuropsychological functioning: a critical review. I Clin Exp Neuropsychol 2012;34: 35-56.

20. Stoodley CJ. The cerebellum and cognition: evidence from functional imaging studies. Cerebellum 2012;11:352-365.

21. Bellebaum C, Daum I. Cerebellar involvement in executive control. Cerebellum 2007;6:184-192.

22. Robinson S, Cohen AR. Cowden disease and Lhermitte-Duclos disease: an update. Case report and review of the literature. Neurosurg Focus 2006; 20:E6.

23. Vinchon M, Blond S, Lejeune JP, et al. Association of Lhermitte-Duclos and Cowden disease: report of a new case and review of the literature. $J$ Neurol Neurosurg Psychiatr 1994;57:699-704.

24. Robinson S, Cohen AR. Cowden disease and Lhermitte-Duclos disease: characterization of a new phakomatosis. Neurosurgery 2000;46:371-383. 